\title{
Factors Influencing Cognitive Dysfunction in Korean Patients with Rheumatoid Arthritis
}

\author{
Shin, So Young \\ Associate Professor, College of Nursing, Inje University, Busan, Korea
}

\begin{abstract}
Purpose: This study was conducted to examine the proportion and potential predictors of cognitive dysfunction in Korean patients with rheumatoid arthritis (RA). Methods: Patients with RA enrolled in this study during follow-up checkups at one general hospital in Korea. A trained research nurse examined participants using a variety of physical, psychosocial, and biological measurements. The Computerized Neuropsychological Function Test, consisting of 16 indicators encompassing a variety of cognitive subdomains, evaluated each participant's cognitive function. Participants were categorized as "impaired" if they performed one standard deviation (SD) below the age-based population standards on each test. Adding the converted scores (range 0 16) generated the total cognitive function score. Multiple linear regression analyses were conducted to identify the significant factors influencing cognitive dysfunction. Results: Sixty-five participants with a $\mathrm{M} \pm \mathrm{SD}$ age of $62.3 \pm 10.45$ years were included in this study. The total cognitive function score was $9.69 \pm 3.50$ (range 2 16). The final multivariate regression model was statistically significant; the model explained $32.7 \%$ of cognitive dysfunction variance. Even after controlling for disease severity, glucocorticoid use, depression, and functional limitations, education level ( $\beta=-.35, p=.005)$, income $(\beta=-.26, p=.041)$, and cardiovascular disease risk factors $(\beta=.28, p=.016)$ significantly influenced cognitive dysfunction. Conclusion: The findings suggest that future studies regarding which etiological variables contribute to cognitive impairment and how to best assess cognitive function in RA patients must occur due to the high risk potential of cognitive dysfunction.
\end{abstract}

Key Words: Cognitive dysfunction; Neuropsychological tests; Cardiovascular disease; Rheumatoid arthritis

\section{INTRODUCTION}

Rheumatoid arthritis (RA) is a chronic inflammatory multisystem autoimmune disorder that causes physical symptoms including pain, joint stiffness, joint swelling, and physical disability as well as psychological symptoms including fatigue and depression [1]. Due to the prolonged life span and the nature of disease chronicity, the number of Korean older adults living with RA-related adverse outcomes including functional decline and high medical costs has increased over time [2]. Identifying the risk factors that could worsen the functional status of this population is a priority task for developing effective strategies to reduce these outcomes.

For patients with chronic diseases including RA, unim- paired cognitive capacity is critical for daily functioning, treatment compliance, and self-management $[3,4]$. Disease-specific features of RA such as systemic chronic inflammation [5] or increased comorbid cardiovascular disease (CVD) risk [6] may be closely linked to neurocognitive dysfunction in RA patients. However, evidence of brain involvement in RA is very rare or even controversial, and very little is known about the pathogenic mechanisms of cognitive decline in persons with RA.

Four previous studies have assessed a range of cognitive subdomains in well-defined groups of RA patients using an inclusive neuropsychological test battery and reported the proportion of cognitive dysfunction in RA patients to be $30 \sim 71 \%[7,8]$. In particular, Shin et al. [4] reported the remarkable finding that along with less edu-

\footnotetext{
Corresponding author: Shin, So Young https://orcid.org/0000-0001-5444-7324

College of Nursing, Inje University, 75 Bokji-ro, Busanjin-gu, Busan 47392, Korea.

Tel: +82-51-890-6934, Fax: +82-51-896-9840, E-mail: syshin@inje.ac.kr

Received: Apr 23, 2020 / Revised: Jun 11, 2020 / Accepted: Jul 10, 2020

This is an open access article distributed under the terms of the Creative Commons Attribution Non-Commercial License (http://creativecommons.org/licenses/ by-nc/3.0), which permits unrestricted non-commercial use, distribution, and reproduction in any medium, provided the original work is properly cited.
} 
cation and low income, which are commonly known sociodemographic factors contributing to cognitive decline, steroid use and increased CVD risk factors, which are specific features of RA, appear to be significant factors that might affect cognitive dysfunction in RA patients. In addition, Hamed et al. [9] reported that the disease-specific process of RA, inflammation and demyelination in the nervous system, might be associated with neurocognitive abnormalities indicated by the presence of brain involvement biomarkers. The findings of these few studies are meaningful in that they place emphasis on the possible cognitive issue and its potential disease-related contributors including disease activity or severity, glucocorticoid use, and CVD risk in persons with RA.

Cognitive dysfunction has been reported as one of the significant risk factors that might severely disrupt functioning and welfare in older adults with chronic health problems $[10,11]$. Because RA is a chronic, irreversible illness, older adults with RA may experience accelerated strain of cognitive decline attributed to both age and disease [4]. Evaluating a wide range of cognitive subdomains in RA patients may encourage appreciating the strain of cognitive problems in these patients and developing effective strategies to decrease damaging consequences on their health [4]. A preliminary study aiming to comprehensively assess cognition in Korean RA patients was conducted by the author and colleagues [12]. The authors observed worse outcomes in cognitive functioning in Korean RA patients compared to the aforementioned studies that were conducted in western countries. The author extended the psychometric analysis to precisely identify the complex mechanism of cognitive deficits in this population. The aim of this study was to examine the proportion of cognitive dysfunction in Korean patients with RA and to investigate the specific factors that were associated with cognitive dysfunction in these patients.

\section{METHODS}

\section{Design}

This is a cross-sectional, correlational study based on the secondary analysis of the data collected for a study conducted by Yoon et al. [12].

\section{Sample and Setting}

Detailed study methods including sample, setting, and measures are described elsewhere [12]. Briefly, the participants of this study included individuals with RA re- cruited during follow-up checkups at one general hospital in Korea. Patients that were unavailable for the whole duration of the assessments due to neuropsychological diseases and visual problems were excluded. After providing signed consents, participants were assessed by a trained research nurse with a range of physical, psychosocial, and biological measurements in a private room. The research nurse repeatedly explained the test-taking methods before and during the tests and helped the participants who needed assistance in taking computerized neuropsychological tests. The duration of all assessments was approximately 60 90 minutes. Finally, 65 participants who provided complete data for all outcome measures and covariates of interest were included in this study. The research protocol was approved by the University Institutional Review Board (No. 2-1041024-AB-N-01020140829-HR-080), and all participants gave their informed consent to participate.

\section{Measures}

\section{1) Cognitive function}

Cognitive function was assessed using the Computerized Neuropsychological Function Test (CNT) developed by Maxmedica Inc. and Ha et al. [13]. The CNT is a computerized version of neuropsychological tests that have been administered via paper and pencil. The most current version is the CNT V4.0 including 17 subtests that assess memory, attention, visuo-motor coordination, and higher-order cognitive capacity $[14,15]$. The subtests of the CNT V4.0 are consistent with the American College of Rheumatology neuropsychological battery which is primarily designed for use in a comparable rheumatic condition, systemic lupus erythematosus, and is deemed reliable and valid [16]. Six tests were selected including Visual Span Test (visuo-spatial memory), Verbal Learning Test (language memory), Visual Continuous Performance test (continuous attention), Word-Color Test (selective attention), Cart Sorting test (executive function), and Trail Making Test (visuo-motor coordination). The duration of the six tests was approximately $40 \sim 60$ minutes.

T-scores based on age-stratified population norms and 16 neuropsychological indices were derived from the scores of the neurological tests. Using conventional cutoff points, participants were classified as "impaired" if they performed one standard deviation (SD) below the age-stratified population norms for each cognitive test [16]. The total cognitive function score was generated by summing the number of tests ranging from 0 to 16 (higher scores=greater impairment). The Cronbach's $\alpha$ was .78 in this study. 


\section{2) Covariates}

Sociodemographic and disease-related characteristics were assessed based on self-reported information. Blood samples for the measurement of $\mathrm{C}$-reactive protein (CRP), total cholesterol, high density lipoprotein (HDL) cholesterol, and low density lipoprotein (LDL) cholesterol were collected during the visit and sent to a laboratory for analysis. Blood pressure as well as height and weight to calculate the body mass index were also measured during the study visit.

The severity of RA was assessed using the Disease Activity Score-28 (DAS-28) [17] which has been tested as a valid and reliable instrument in many RA studies [4]. It is calculated according to four components: tender joint count, swollen joint count, global health score on a visual analog scale, and the laboratory parameter of CRP. The total score ranges from 0 to 9.4 , with higher scores indicating greater disease activity [17].

Cardiovascular disease (CVD) risk factor scores were generated based on variables in the CVD risk score profiles from the Framingham heart study [18]. Scores were calculated as the total number of the following CVD risk factors that were present: hypertension, diabetes mellitus, systolic blood pressure $>140 \mathrm{mmHg}$, antihypertensive medication use, HDL cholesterol $<60 \mathrm{mg} / \mathrm{dL}$, LDL cholesterol $>130 \mathrm{mg} / \mathrm{dL}$, current smoking, and obesity (body mass index $>25$ ). CVD risk factor scores ranges from 0 to 8 , with higher scores indicating greater CVD risk.

Depression was assessed using the Korean version of the short-form Geriatric Depression Scale (GDS) translated and validated by Bae and Cho [19]. It comprises 15 questions requiring participants to respond with a yes or no regarding how they felt over the past week. The total score ranged from 0 to 15 , with higher scores indicating greater depression [19].

Functional limitations were assessed using the Korean version of the Health Assessment Questionnaire (HAQ) translated and validated by Bae et al. [20]. It comprises 20 items covering physical actions in eight domains: dressing and grooming, arising, eating, walking, personal hygiene, reaching, gripping, and outdoor activities. The HAQ score ranged from 0 to 3, with higher scores reflecting greater functional limitations [19].

\section{Statistical Analyses}

Sociodemographic and disease-related characteristics were analyzed using descriptive statistics. The degree of variables of interest was described as $\mathrm{M} \pm \mathrm{SD}$ (range). Multiple linear regression analyses were used to iden- tify the significant factors related to cognitive dysfunction and included variables that were associated with cognitive dysfunction in previous studies on individuals with chronic health conditions. Education level, family income, disease activity, oral glucocorticoid use, CVD risk factor scores, depression, and functional limitations were assessed to be the potential predictors of cognitive dysfunction. The limit for significance was set at two-tailed $=0.05$. All analyses were conducted using the IBM SPSS Statistics, version 25.0 .

\section{RESULTS}

\section{Characteristics of Participants}

Participants' characteristics are presented in Table 1. The average age of 65 participants was $62.3 \pm 10.45$ (42 86) years. $84.6 \%$ were female and $87.7 \%$ were married. $76.2 \%$ had less than 12 years of education and $40.0 \%$ had less than 1,500,000 Korean Won of family income. The DAS-28

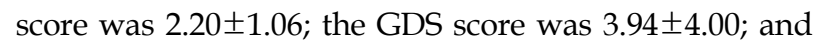
the HAQ score was $0.29 \pm 0.53$.

Table 1. Characteristics of Participants

$(N=65)$

\begin{tabular}{lcc}
\hline Variables & $\begin{array}{c}\mathrm{n}(\%) \text { or } \\
\mathrm{M} \pm \mathrm{SD}\end{array}$ & Range \\
\hline Age (year) & $62.3 \pm 10.45$ & $42 \sim 86$ \\
Female & $55(84.6)$ & \\
Married & $57(87.7)$ & \\
Education level 12 (year) & $48(76.2)$ & \\
Family income $<150$ & $26(40.0)$ & \\
$\quad$ (10,000 Korean Won) & & \\
Current steroid use & $43(66.2)$ & \\
\hline Duration of RA (year) & $10.10 \pm 8.76$ & $0.17 \sim 42.00$ \\
\hline Severity of RA (DAS-28) & $2.20 \pm 1.06$ & $0.51 \sim 5.95$ \\
\hline CVD risk factors & $2.31 \pm 1.51$ & $0 \sim 6$ \\
\hline Depression (GDS) & $3.94 \pm 4.00$ & $0 \sim 14$ \\
\hline Functional limitations (HAQ) & $0.29 \pm 0.53$ & $0.00 \sim 2.50$ \\
\hline
\end{tabular}

RA=Rheumatoid arthritis; DAS-28=Disease Activity Score-28; $\mathrm{CVD}=$ Cardiovascular disease; GDS=Geriatric Depression Scale; $\mathrm{HAQ}=$ Health Assessment Questionnaire.

\section{Cognitive Dysfunction of Participants}

The CVD risk factors score was $2.31 \pm 1.51$ (Table 1). The total cognitive function score was $9.69 \pm 3.50$ (Table 2). The percentage of participants who were categorized as 
Table 2. Number of Participants with Cognitive Impairment by Each Neuropsychological Test Domain

\begin{tabular}{|c|c|c|}
\hline Domain & Categories & $\mathrm{n}(\%)$ \\
\hline Visuo-spatial learning and memory impairment & $\begin{array}{l}\text { Visual span test forwards } \\
\text { Visual span test backwards }\end{array}$ & $\begin{array}{l}46(70.8) \\
35(53.8)\end{array}$ \\
\hline Verbal learning and memory impairment & $\begin{array}{l}\text { Verbal learning test first attempt } \\
\text { Verbal learning test second attempt } \\
\text { Verbal learning test short delay free recall } \\
\text { Verbal learning test long delay free recall }\end{array}$ & $\begin{array}{l}33(50.8) \\
45(69.2) \\
43(66.2) \\
54(83.1)\end{array}$ \\
\hline Continuous attention impairment & $\begin{array}{l}\text { Visual continuous performance test correct response } \\
\text { Visual continuous performance test omission error } \\
\text { Visual continuous performance test commission error }\end{array}$ & $\begin{array}{l}15(23.1) \\
15(23.1) \\
35(53.8)\end{array}$ \\
\hline Selective attention impairment & $\begin{array}{l}\text { Word-color test-word } \\
\text { Word-color test-color } \\
\text { Word-color test discordance-word } \\
\text { Word-color test discordance-color }\end{array}$ & $\begin{array}{l}45(69.2) \\
53(81.5) \\
46(70.8) \\
60(92.3)\end{array}$ \\
\hline Visuo-motor coordination impairment & $\begin{array}{l}\text { Trail making test } \mathrm{A} \\
\text { Trail making test } \mathrm{B}\end{array}$ & $\begin{array}{l}40(61.5) \\
42(64.6)\end{array}$ \\
\hline Higher-order cognitive capacity impairment & Card sorting test & $23(35.4)$ \\
\hline At least $5(25 \%)$ of cognitive tests impaired & & $61(93.8)$ \\
\hline $\begin{array}{l}\text { Total cognitive function score } \\
(\mathrm{M} \pm \mathrm{SD} \text {, range })\end{array}$ & & $\begin{array}{l}9.69 \pm 3.50 \\
(2.00 \sim 16.00)\end{array}$ \\
\hline
\end{tabular}

cognitively impaired on each test domain of the CNT ranged from $23.1 \%$ (continuous attention test) to $92.3 \%$ (selective attention test). All participants were identified as cognitively impaired on at least 2 or more out of the 16 subtests. The ratio of persons identified as cognitively impaired on a quarter of the total subtests (4 or more out of the 16 subtests) was $93.8 \%$ (Table 2).

\section{Factors Associated with Cognitive Dysfunction of Participants}

Before performing the multivariate regression analysis, the assumptions of the analysis were examined. First, the Durbin-Watson value was 2.09, indicating that the variables were not autocorrelated. The tolerance was $<1.0(.56 \sim .90)$ and the variance inflation factor was $<10.0$ (1.12 1.80), demonstrating there were no problems associated with multicollinearity. Moreover, the normality and homoscedasticity of residuals were tested using histograms, normal probability plots, and scatter plots, and the results showed that the residuals satisfied the assumptions.

Education level, family income, severity of RA, oral glucocorticoid use, the number of CVD risk factors, depression, and functional limitations were included in multivariate regression models (Table 3 ). These seven variables accounted for $32.7 \%$ of the variance in cognitive dys- function $(\mathrm{F}=5.44, p<.001)$. Of the disease-related variables, the CVD risk factor score was significantly associated with cognitive dysfunction $(\beta=.28, p=.016)$; education level $(\beta=-.35, p=.005)$ and family income $(\beta=-.26, p=.041)$ were the only sociodemographic variables associated with cognitive dysfunction (the result was already adapted for age) even after controlling for other covariates in this cohort.

\section{DISCUSSION}

This study explored the proportion and potential predictors of cognitive dysfunction in Korean patients with RA. The ratio of persons who were identified as cognitively impaired on each test was from $23.1 \%$ to $92.3 \%$. The majority of the participants were identified as cognitively impaired on four or more tests. In multivariate models, education level and family income were the sociodemographic factors significantly influenced cognitive dysfunction. Only an accumulative number of CVD risk factors appeared as disease-related clinical indicators significantly influenced cognitive dysfunction in this population.

The most commonly influenced cognitive subdomains were verbal learning and memory $(83.1 \%)$ and selective attention $(92.3 \%)$. These results are analogous to those of previous studies. Bartolini et al. [7] reported that 38 71\% 
Table 3. Factors Influencing Cognitive Impairment

\begin{tabular}{lccc}
\hline Variables & $\beta$ & $t$ & $p$ \\
\hline Education level (year) & -.35 & -2.96 & .005 \\
Family income (Korean Won) & -.26 & -2.10 & .041 \\
Severity of RA (DAS-28) & .01 & 0.06 & .951 \\
Current steroid use & .03 & 0.30 & .766 \\
CVD risk factors & .28 & 2.50 & .016 \\
Depression (GDS) & .01 & 0.02 & .988 \\
Functional limitations (HAQ) & -0.73 & .467 \\
\end{tabular}

RA=Rheumatoid arthritis; DAS-28=Disease Activity Score-28; CVD=Cardiovascular disease; GDS=Geriatric Depression Scale; HAQ=Health Assessment Questionnaire.

of their RA participants were cognitive impaired with worse results in visuo-spatial and planning functions. Appenzeller et al. [8] observed cognitive dysfunction in $30 \%$ of the RA group, with poorer outcomes in verbal fluency and episodic memory. Shin et al. [4] observed cognitive dysfunction mostly in visuo-spatial learning/memory $(28 \%)$ and design fluency $(29 \%)$ in their RA group. One third of their subjects were classified as cognitively impaired on a quarter of the total subtests (4 or more out of the 16 subtests) [4]. Hamed et al. [9] observed that $71 \%$ of RA patients had cognitive deficits. When compared to healthy controls, RA patients showed worse outcomes in 11 subtests assessing 4 subdomains of cognition (verbal function, visuo-spatial organization, memory, and judgement/problem-solving)[9]. Although direct comparisons across studies may be unfeasible due to dissimilar classifying standards of cognitive dysfunction and various evaluation methodology used, these results do underscore the significant cognitive problems of RA patients that may in turn disrupt their daily functioning and possibly make it more difficult to manage health conditions.

Our participants had much poorer outcomes in cognitive dysfunction compared to participants in the aforementioned studies. One possible explanation for this result could be the ethnic difference and cultural characteristics of Korean older adults. Because Korean society has been strongly influenced by the Confucian culture, Korean older adults tend to endure their symptoms and are reluctant to visit hospitals to take formal cognitive tests [12]. Therefore, when administering formal neuropsychological assessments in an unfamiliar hospital environment, Koreans may have different and somewhat severe cognitive problems that could have been less severe impairment if identified earlier in comparison with persons in western countries.
Furthermore, the results of cognitive function evaluated by objective neurocognitive tests may be considerably distinct from those assessed by subjective cognitive measures [12]. Further studies with more diverse and representative groups of population with well-standardized assessments are needed to identify the complicated mechanism of cognitive dysfunction in RA patients.

Commonly well-known sociodemographic risk factors of cognitive dysfunction in persons with or without chronic diseases include lower levels of education and family income $[21,22]$. Similar to the previous studies on cognitive function in chronic problems, both less education and lower family income were identified as significant factors influencing cognitive dysfunction of the participants in this study. In addition, CVD risk factors appeared to be the most significant factor associated with cognitive dysfunction in Korean RA patients. This finding indicates that the participants with an increased number of CVD risk factors are more likely to be cognitively impaired. This result is in accordance with the study finding by the previous studies that CVD risk factors are known contributors of cognitive decline [23]. Specifically for RA patients, Shin et al. [4] reported the same results that CVD risk factors as a significant predicting factor of cognitive impairment. This might be due to the fact that accelerated atherosclerotic changes caused by systemic chronic inflammation may escalate the risk of cardiovascular comorbidity $[6,24]$. Growing evidence suggests increased cardiovascular risk in RA patients which is analogous to that of type 2 diabetes and emphasizes that RA is a new, independent CVD risk factor which should be carefully managed [24]. In this respect, accompanied CVD risk factors including hypertension, diabetes mellitus, hyperlipidemia, obesity, or current smoking may increase 
the occurrence of cognitive dysfunction in RA patients. Further studies are necessary to identify the exact mechanism between RA and increased CVD risk in causing cognitive decline.

Glucocorticoid use, which is a common treatment of RA, did not appear to be a significant contributor of cognitive dysfunction in this study. This finding is in contrast with a study result by Shin et al. [4] who reported that patients with even low dose of steroid use were more likely to have cognitive dysfunction. Glucocorticoid therapy has been reported to be associated with memory deficits and to possibly have cumulative and ongoing impact on hippocampal functions [25]. Although there seems to be a possible and significant association between glucocorticoid medication and cognitive function in RA patients, the exact mechanism of this association is unclear. Further studies to identify the specific influence of glucocorticoid use on neurocognitive functioning in RA patients are necessary.

In previous studies, RA activity or severity has been linked with cognitive dysfunction [26]. Clinical symptomatic features of RA including chronic pain and psychological distress were related with cognitive dysfunction $[27,28]$. However, these factors were not significant predictors of cognitive dysfunction in this study. The possible reasons of these conflicting findings may be either the relatively low levels of disease activity (mean DAS-28 score $=2.20$ [range $=0.51 ~ 5.95]$ ) and depressive mood (mean GDS score $=3.94$ [range $=0 \sim 14] ; 21.6 \%$ of participants appeared to be depressive, i.e., GDS score 8 ) in this group or the distinctive assessment methods. The aforementioned sociocultural characteristics of Korean older adults may also affect the study results. Korean older adults are inclined to bear their symptoms, especially psychological or cognitive symptoms, and are reluctant to take tests to be officially diagnosed by a health professional [12]. Strict social stigma to neuropsychiatric diseases perhaps constrains Korean older adults from openly reporting their psychological distress. Further studies should be conducted to verify the exact influence of these factors on cognitive dysfunction in persons with RA.

There are some limitations in this study. Only small numbers of individuals who were relatively healthy enough to finish a range of tests possibly may limit the generalizability of study findings. Although the required test-taking skill for the computerized neurocognitive assessments used in this study was clicking mouse buttons, which was simple and easy, and the research nurse helped the participants who needed any kind of assistance, the lack of familiarity with computers might influence the cognitive test results.

Despite some limitations, this study has several strengths. The classification standards of cognitive dysfunction in this study are analogous in rigor to those in other studies on rheumatic diseases $[4,16]$. An extensive range of cognitive dysfunction in RA can be evaluated, as even mild cognitive problems can interrupt daily functioning, medication adherence, and self-care [4]. This study results can be even more significant in that the participants' current conditions are relatively healthy with fewer disease-related symptoms.

To my knowledge, this is the first study that provided rich information on cognitive subdomains in Korean patients with RA and possible predictors of cognitive dysfunction using a set of computerized neuropsychological tests. As a computerized neuropsychological test is automated and standardized, a subject's cognitive function can be assessed objectively and precisely with less time and cost, and minimal external influence (e.g., environment, administrators) [12]. Test results can be easily and rapidly calculated, analyzed, and compared with norms [29]. Therefore, by using a variety of computerized neuropsychological tests, a patient's cognitive function can be explored comprehensively.

This study has noteworthy implications for gerontological health care. The findings of this study underscore the potential significance of applying a computerized cognitive function test in practice as a meaningful and necessary strategy for older adults with RA who may have an enhanced risk of cognitive decline because of aging and disease process. In both clinical and research settings, bedside mental status screening tests have been widely used for assessing cognitive function due to their accessibility and efficiency. However, a few studies have reported the inconsistency between self-reported symptoms and objectively measured results of cognitive dysfunction in diverse populations, including RA patients [12]. Because older adults have distinctive features related to aging process such as hearing loss or geriatric depression which may cause confusion with cognitive dysfunction, accurate evaluation of cognitive function is even more challenging. Future studies should focus on identifying the best assessment strategy of cognitive function in this population.

In practice, health care professionals should prudently evaluate the cognitive function of older patients with RA, particularly those with comorbid CVD risk. The increased CVD risk, which is a renowned aggravating factor of cognitive dysfunction in the general population, has been substantially noticed in RA. Adequate CVD treatment 
and prevention may advance cognitive function or hinder the occurrence of cognitive alterations [4]. The American Heart Association stated that hypertension may stimulate cognitive decline pathology by ischemic injury to white matter regions which is crucial for cognitive function [30]. Further studies identifying both the impact of CVD in causing cognitive decline and the role of management and prevention of CVD in relieving these neuropsychiatric alterations are necessary.

\section{CONCLUSION}

The present study explored the proportion and possible predicting variables of cognitive dysfunction in Korean patients with RA. A considerable number of RA patients were observed to have cognitive dysfunction in this study. Less education, lower family income, and increased accumulative number of CVD risk factors were the significant factors affecting cognitive impairment in persons with RA. The study results indicate the need for reinforcing the potential health problems related to cognitive dysfunction in older adults with RA. Further investigation on what etiological variables contribute to cognitive impairment and how to best assess cognitive impairment in RA should be conducted.

\section{CONFLICTS OF INTEREST}

The author declared no conflict of interest.

\section{AUTHORSHIP}

Study conception and design acquisition - SYS; Data analysis and interpretation -SYS; Drafting and critical revision of the manuscript - SYS; Final approval - SYS.

\section{ACKNOWLEDGEMENT}

This study was supported by the 2018 Inje University research grant (No.20180148).

I gratefully acknowledge Dr. Yoon, B.Y. and Dr. Lee, J.H. for their support on data collection.

\section{REFERENCES}

1. Jeffery RC. Clinical features of rheumatoid arthritis. Medicine. 2014;42(5):231-6. https://doi.org/10.1016/j.mpmed.2014.02.011

2. Sung YK, Cho SK, Choi CB, Bae SC. Prevalence and incidence of rheumatoid arthritis in South Korea. Rheumatology International. 2013;33(6):1525-32. https://doi.org/10.1007/s00296-012-2590-2
3. Abeare CA, Cohen JL, Axelrod BN, Leisen JCC, Mosley-Williams A, Lumley MA. Pain, executive functioning, and affect in patients with rheumatoid arthritis. Clinical Journal of Pain. 2010;26(8):683-9.

https://doi.org/10.1097/AJP.0b013e3181ed1762

4. Shin SY, Katz P, Wallhagen M, Julian L. Cognitive impairment in persons with rheumatoid arthritis. Arthritis Care \& Research. 2012;64(8):1144-50. https://doi.org/10.1002/acr.21683

5. Wallin K, Solomon A, Kåreholt I, Tuomilehto J, Soininen H, Kivipelto M. Midlife rheumatoid arthritis increases the risk of cognitive impairment two decades later: a population-based study. Journal of Alzheimer's Disease. 2012;31(3):669-76. https://doi.org/10.3233/JAD-2012-111736

6. Gkaliagkousi E, Gavriilaki E, Doumas M, Petidis K, Aslanidis $\mathrm{S}$, Stella D. Cardiovascular risk in rheumatoid arthritis: pathogenesis, diagnosis, and management. Journal of Clinical Rheumatology. 2012;18(8):422-30. https://doi.org/10.1097/RHU.0b013e31827846b1

7. Bartolini M, Candela M, Brugni M, Catena L, Mari F, Pomponio $G$, et al. Are behaviour and motor performances of rheumatoid arthritis patients influenced by subclinical cognitive impairments? a clinical and neuroimaging study. Clinical and Experimental Rheumatology. 2002;20(4):491-7.

8. Appenzeller S, Bertolo MB, Costallat LT. Cognitive impairment in rheumatoid arthritis. Methods and Findings in Experimental and Clinical Pharmacology. 2004;26(5):339-43. https://doi.org/10.1358/mf.2004.26.5.831324

9. Hamed SA, Selim ZI, Elattar AM, Elserogy YM, Ahmed EA, Mohamed HO. Assessment of biocorrelates for brain involvement in female patients with rheumatoid arthritis. Clinical Rheumatology. 2012;31(1):123-32. https://doi.org/10.1007/s10067-011-1795-1

10. Auyeung TW, Kwok T, Lee J, Leung PC, Leung J, Woo J. Functional decline in cognitive impairment-the relationship between physical and cognitive function. Neuroepidemiology. 2008;31 (3):167-73. https://doi.org/10.1159/000154929

11. Raji MA, Kuo YF, Snih SA, Markides KS, Peek MK, Ottenbacher KJ. Cognitive status, muscle strength, and subsequent disability in older Mexican Americans. Journal of the American Geriatrics Society. 2005;53(9):1462-8. https://doi.org/10.1111/j.1532-5415.2005.53457.x

12. Yoon BY, Lee JH, Shin SY. Discrepancy between subjective and objective measures of cognitive impairment in patients with rheumatoid arthritis. Rheumatology International. 2017; 37(10):1635-41. https://doi.org/10.1007/s00296-017-3806-2

13. Ha KS, Kwon JS, Lyoo IK, Kong SW, Lee DW, Youn T. Development and standardization process, and factor analysis of the computerized cognitive function test system for Korea adults. Journal of Korean Neuropsychiatric Association. 2002; 
41(3):551-62.

14. Kim YH, Ko MH, Seo JH, Park SH, Kim GS, Jang EH, et al. Effect of computer-assisted cognitive rehabilitation program for attention training in brain injury. Journal of the Korean Academy of Rehabilitation Medicine. 2003;27(6):830-9.

15. Park JI, Lee HJ, Cho HJ. Neuropsychological functioning in detoxified alcoholics by Seoul Computerized Neurocognitive Function Test. Journal of Korean Neuropsychiatric Association. 2004;43(5):570-6.

16. Kozora E, Ellison MC, West S. Reliability and validity of the proposed American College of Rheumatology neuropsychological battery for systemic lupus erythematosus. Arthritis Care \& Research. 2004;51(5):810-8. https://doi.org/10.1002/art.20692

17. Prevoo ML, Van'T Hof MA, Kuper HH, Van Leeuwen MA, Van De Putte LB, Van Riel PL. Modified disease activity scores that include twenty-eight-joint counts. Development and validation in a prospective longitudinal study of patients with rheumatoid arthritis. Arthritis \& Rheumatology. 1995;38(1): 44-8. https://doi.org/10.1002/art.1780380107

18. D'Agostino RB, Sr., Vasan RS, Pencina MJ, Wolf PA, Cobain M, Massaro JM, et al. General cardiovascular risk profile for use in primary care: the Framingham Heart Study. Circulation. 2008;117(6):743-53. https://doi.org/10.1161/CIRCULATIONAHA.107.699579

19. Bae JN, Cho MJ. Development of the Korean version of the Geriatric Depression Scale and its short form among elderly psychiatric patients. Journal of Psychosomatic Research. 2004; 57(3):297-305.

https://doi.org/10.1016/j.jpsychores.2004.01.004

20. BaeSC, Cook EF, Kim SY. Psychometric evaluation of a Korean Health Assessment Questionnaire for clinical research. Journal of Rheumatology. 1998;25(10):1975-9.

21. Lee S, Kawachi I, Berkman LF, Grodstein F. Education, other socioeconomic indicators, and cognitive function. American Journal of Epidemiology. 2003;157(8):712-20. https://doi.org/10.1093/aje/kwg042

22. Cagney KA, Lauderdale DS. Education, wealth, and cognitive function in later life. The Journals of Gerontology. Series B, Psychologcial Sciences and Social Sciences. 2002;57(2):163-72. https://doi.org/10.1093/geronb/57.2.P163

23. Singh-Manoux A, Sabia S, Lajnef M, Ferrie JE, Nabi H, Britton $\mathrm{AR}$, et al. History of coronary heart disease and cognitive performance in midlife: the Whitehall II study. European Heart Journal. 2008;29(17):2100-7. https://doi.org/10.1093/eurheartj/ehn298

24. Nurmohamed MT. Cardiovascular risk in rheumatoid arthritis. Autoimmunity Reviews. 2009;8(8):663-7. https://doi.org/10.1016/j.autrev.2009.02.015

25. Coluccia D, Wolf OT, Kollias S, Roozendaal B, Forster A, de Quervain DJF. Glucocorticoid therapy-induced memory deficits: acute versus chronic effects. Journal of Neuroscience. 2008;28(13): 3474-8. https://doi.org/10.1523/JNEUROSCI.4893-07.2008

26. Gimeno D, Marmot MG, Singh-Manoux A. Inflammatory markers and cognitive function in middle-aged adults: the Whitehall II study. Psychoneuroendocrinology. 2008;33(10): 1322-34. https://doi.org/10.1016/j.psyneuen.2008.07.006

27. Geda YE, Roberts RO, Knopman DS, Petersen RC, Christianson TJ, Pankratz VS, et al. Prevalence of neuropsychiatric symptoms in mild cognitive impairment and normal cognitive aging: population-based study. Archives of General Psychiatry. 2008;65(10):1193-8.

https://doi.org/10.1001/archpsyc.65.10.1193

28. Ganguli M. Depression, cognitive impairment and dementia: why should clinicians care about the web of causation? Indian Journal of Psychiatry. 2009;51(Suppl1):S29-34.

29. Gur RC, Ragland JD, Moberg PJ, Bilker WB, Kohler C, Siegel $\mathrm{SJ}$, et al. Computerized neurocognitive scanning: II. the profile of schizophrenia. Neuropsychopharmacology. 2001;25(5): 777-88. https://doi.org/10.1016/s0893-133x(01)00279-2

30. Iadecola C, Yaffe K, Biller J, Bratzke LC, Faraci FM, Gorelick $\mathrm{PB}$, et al. Impact of hypertension on cognitive function: a scientific statement from the American Heart Association. Hypertension. 2016;68(6):e67-94. https://doi.org/10.1161/HYP.0000000000000053 